\title{
El impacto de las remesas en Guatemala ¿Alivio a la pobreza o factor de desarrollo?
}

\author{
Camille Barre $^{1}$
}

Reporte de caso recibido el 30/09/2010 y aprobado el 10/12/2010

\section{Resumen}

Con su numerosa emigración hacia Estados Unidos, Guatemala hace parte los principales países receptores de remesas en América latina. Frente a este fenómeno, presente en toda Latinoamérica, políticos, académicos y organizaciones internacionales se han interesado en el impacto que tienen las remesas para el desarrollo de los países de la región. Este artículo estudia el caso de Guatemala y busca mostrar qué consecuencias económicas y sociales generan estas transferencias de dinero en la sociedad de Guatemala, país con significativos índices de emigración a los Estados Unidos. Para lograr tal objetivo, el presente artículo caracteriza los emigrantes guatemaltecos que envían remesas, estudia el perfil de los hogares receptores de las mimas, y la forma cómo estas son utilizadas. Posteriormente, se muestra que las remesas representan una ayuda significativa para los hogares más pobres, permitiéndoles aumentar el consumo de bienes de primera necesidad y tener acceso a educación y salud. A su vez se observa cuáles son los factores que permiten convertir las remesas en actividades productivas que contribuyen al desarrollo del país, pero también se advierte sobre las limitantes en el uso de estos recursos. Por último, se mostrará la posición del Estado guatemalteco frente a la emigración de sus ciudadanos, las remesas y las políticas públicas formuladas en relación a éstos dos fenómenos.

Palabras clave: Migración internacional, remesas, desarrollo, pobreza

\begin{abstract}
Since remittances are a common phenomenon in Latin America,, politicians, academics and international organizations have been interested to better understand the impact of remittances on development in this part of the world. This article shows the economic and social consequences of these financial transfers in the case of Guatemala, which, because of

1 Camille Barre es estudiante de Maestría en Relaciones Internacionales con énfasis en Seguridad Internacional, en el Instituto de Ciencias Políticas de Paris (Sciences Po), donde, en el 2010 ,se graduó en Ciencias Políticas. En el 2009, realizó un intercambio académico en la Universidad Icesi, en el departamento de Estudios Políticos.
\end{abstract}


a significant emigration to the United States, is one of the key countries to receive remittances. In order to comply with its objective, the paper describes Guatemalan emigrants who send remittances, the general profile of households which receive those transfers and the way their money is spent. The paper argues that remittances constitute a significant help to poor families as they allow them to increase their basic consumption as well as their access to education and health. However, the impact of remittances on national development remains unclear, since it is still necessary to better establish not only which factors favor the use of remittances for productive activities, but also the limitation of the process in question. Finally, this paper discusses the position of the Guatemalan State with regards to its citizen's emigration and the issue of public policies on migration and remittances.

Keywords: International migration, remittance, development, poverty.

\section{Introducción}

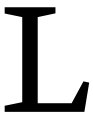

a guerra civil en Guatemala provocó la migración de miles de refugiados políticos a países vecinos, teniendo como principal destino Estados Unidos. $\mathrm{Si}$ bien es cierto que la guerra terminó en el 1996, las hostilidades y tensiones políticas no cesaron en el país,la violencia, las desigualdades y los problemas económicos persistieron, estos factores fueron los que presionaron la emigración. Dada su posición geográfica y su frontera con México, Guatemala es un país muy afectado por la migración irregular puesto que numerosos centroamericanos que regresan a su país desde México tienen que pasar por el territorio guatemalteco. La situación económica también es una de las principales causas de emigración. A finales de los años 90 y principios de la década de 2000, la situación económica del país, que ya era precaria, se deterioró todavía más. La desaceleración de la economía de Estados Unidos, principal socio comercial de Guatemala, afectó al país por su alto grado de dependencia con tal economía. Ante todo, la economía guatemalteca se deterioró por el colapso del café, cuyos precios internacionales cayeron, provocando un agravamiento de la situación agraria. Un informe de la Organización Internacional para las Migraciones (OIM) del 2006² comunica que Guatemala ocupa el $117^{\circ}$ lugar en la clasificación de 177 países en términos de desarrollo humano y que tuvo un crecimiento económico bajo en la última década (2.4\%), altamente basado en la agricultura.Además, el país tiene baja cobertura de seguridad social y presenta problemas humanitarios y de seguridad. En el 2003, el Banco Mundial ${ }^{3}$ estimó que el país tenía uno de los índices de desigualdad más altos de América latina, con el $56 \%$ de la población ubicada bajo la línea de pobreza, y el $16 \%$ en situación de extrema pobreza. La pobreza se concentra principalmente en las áreas rurales, donde se encuentra el $81 \%$ de las personas consideradas pobres y el $93 \%$

2 OIM,(2006). Encuesta sobre Remesas 2006.Inversión en Salud y Educación en Cuadernos de Trabajo Sobre Migración, $\mathrm{n}^{\circ} 23$

3 BANCO MUNdial, (2006) Análisis del corredor de remesas Estados Unidos-Guatemala,24 de abril de 2006 
de los extremadamente pobres. Además de concentrarse en la población rural,la pobreza y extrema pobreza afectan de manera desproporcional a la población indígena y a las mujeres. Finalmente, el hecho de que el país sea la última frontera con la NAFTA aumenta la presión migratoria irregular de los guatemaltecos que buscan soluciones económicas en el norte del continente americano. Los emigrantes envían regularmente remesas a sus familiares, las cuales son consideradas por instituciones internacionales como el Banco Mundial como vías posibles de desarrollo para los países beneficiarios. El objetivo de este estudio será, por lo tanto, observar en qué medida las remesas guatemaltecas contribuyen al desarrollo del país en la primera década de siglo XXI. Organizaciones internacionales tales como el Banco Mundial y los Estados tienden a considerar las remesas como una oportunidad de desarrollo para países donde estas transferencias representan una parte importante del PIB.Sin embargo, el presente artículo sostiene que estos actores se basan en una perspectiva económica y no consideran las consecuencias sociales que la emigración masiva y el envío de remesas pueden provocar para el país de origen. Si bien las remesas representan un alivio para los sectores más pobres de Guatemala, no son una manera efectiva de fomentar desarrollo y se puede cuestionar la legitimidad de usar estas transferencias para el desarrollo nacional. De este modo nos preguntaremos si estas transferencias financieras fomentan el crecimiento económico, si reducen los niveles de pobreza y la desigualdad en el país, si ayudan a los hogares receptores a tener más acceso a servicios de salud y educación y si procuran por disminuir el nivel de abandono escolar.Para esto, se observará en un primer momento, el perfil de los emigrantes, de los hogares beneficiarios y el uso que hacen de las remesas. Luego se estudiará el rol que juegan las remesas en el desarrollo del país, y finalmente se presentarán las posturas y acciones del Estado guatemalteco y de otros actores en relación al uso de las remesas para el fomento del desarrollo.

\section{Guatemaltecos en el exterior, hogares en Guatemala y remesas}

Para el año 2006, se estima que las remesas enviadas a hogares en Guatemala representaban 3.425.009.993 dólares ${ }^{4} y$ que el promedio mensual de remesa por hogar era de 309 dólares, por lo cual el monto de remesas enviadas al país es importante para su economía. Según estimaciones del Banguat, un banco guatemalteco, el total de las remesas enviadas a Guatemala representaba en el 2005 el 9.4\% del PIB. ${ }^{5}$

La migración internacional de los guatemaltecos es un fenómeno importante puesto que la proporción de emigrantes en relación a la población guatemalteca crece sin parar y se está acelerando. La tendencia migratoria se aceleró en 1995, luego presentó una baja en 2001 como consecuencia de los atentados del 11 de septiembre, y finalmente volvió a incrementarse de manera continua a partir de 2002. En este año, según un informe de la OIM de 2006, los guatemaltecos en el exterior representaban el $10.5 \%$ de la población. En el 2006, esta cifra pasó al 10.9\%, un porcentaje similar de los colombianos

4 Opcit.

5 Opcit 
que están en el exterior. La gran mayoría de estos emigrantes va a Estados Unidos (98.2\%), donde se encuentra el 96.9\% de los que envían remesas. Las principales ciudades de destino en Estados Unidos son Los Ángeles (34.7\%), New York (10.2\%), Miami (8.1\%),Washington (4.1\%)y Norfolk (3.3\%). Las ciudades donde se instalan los guatemaltecos son las que ya tienen gran presencia de migrantes. En efecto, las redes de contactos cuentan mucho a la hora de emigrar porque tornan la llegada al país y la integración mucho más fácil.

"Dada su posición geográfica y su frontera con México, Guatemala es un país muy afectado por la migración irregular puesto que numerosos centroamericanos que regresan a su país desde México tienen que pasar por el territorio guatemalteco»

Por lo general, los hombres guatemaltecos emigran más que las mujeres, según el informe, debido a que éstas tienden a permanecer más en el país cuidando a sus hijos, y que los riesgos que corren en el traslado ilegal a otros países es mayor para ellas que para los hombres, lo cual torna la decisión de emigrar más difícil. Como lo muestran las cifras, casi todos los emigrantes envían remesas a sus hogares, a diferencia del $6 \%$ que no lo hace. El mismo informe considera que este porcentaje se explica por lo que los hogares también se componen de niños y estudiantes, los cuales no están en capacidad de enviar remesas. El promedio de emigrantes por hogar que recibe remesas sería entonces de 1.3 personas.

La mayoría de los guatemaltecos en el exterior es relativamente joven e indocumentada.El $77.3 \%$ de los emigrantes tiene entre 20 y 44 años, el $17.4 \%$ tiene 45 años o más y el 3.8\% tiene menos de 20 años. La mayoría de los emigrantes guatemaltecos (56.7\%) proviene de áreas rurales. En lo que concierne al nivel de escolaridad de los emigrantes antes de dejar su país de origen, la mayoría no tiene más que el nivel primario. El 50.6\% viajó con algún grado de educación primaria, el 23.5\% no tenía la primaria completa y el $27.1 \%$ había completado la primaria. Muy pocos son los emigrantes que tenían algún grado universitario (1.2\%) y menos aún los que ya eran profesionales (0.6\%). Las razones de emigración son principalmente económicas y se componen en un $67 \%$ por falta de empleo, en el $21.1 \%$ por falta de capacitación para trabajar, y el 6.5\% por el bajo nivel de salario; estos datos revelan que los emigrantes guatemaltecos dejan su país por razones económicas, para encontrar mejores empleos y mayores ingresos con el fin de ayudar a sus familias enviándoles remesas.(OIM, 2006 y Lozano ${ }^{6}$ )

Según el mismo documento de la OIM, en el 2006, una gran mayoría de la población guatemalteca depende de las remesas, el $81.4 \%$ de la población residente en Guatemala recibió remesas mensualmente en ese año. De estas per-

6 LOZANO, Victor (2005). Encuesta Nacional sobre emigración internacional de guatemaltecos 2002-2003 en Población y Salud en Mesoamérica, Vol.2, N², art.4, ene-jun. 2005,pg.20 
sonas, la mayoría reside en áreas rurales (57.2\%) y son mujeres (54.6\%).El 22\% de los beneficiados por las remesas pertenece a la población indígena. Es pertinente considerar el nivel educativo de los que reciben remesas porque se deduce que a mayor nivel de educación, mejores son los empleos y los ingresos de los hogares, y a menor nivel educativo, mayor es la probabilidad que el hogar esté en una situación económicamente precaria. Entre los receptores de remesas, el 39\% no tiene educación primaria completa. La mayoría de los emigrantes guatemaltecos que se van del país y envían remesas son los que pertenecen a hogares con bajos ingresos y se van por razones económicas, puesto que no encuentran soluciones u oportunidades en su propio país. De hecho, sólo el $38 \%$ de los hogares que recibe remesas tiene una cabeza de familia económicamente activa. Los dos sectores de ocupación que más reciben remesas son los que tienen una actividad relacionada al comercio (25.9\%), sea este formal o informal,y a la agricultura (24\%).

Los principales canales de envío de remesas son tanto formales como informales y se dividen entre las transferencias electrónicas (77.7\%) como Western Unión, Money Gram y Vigo, luego a través de Money Order (12.7\%), también por cuentas bancarias $(6.6 \%)$ y finalmente a través de viajeros frecuentes (3\%).

Las remesas recibidas por los hogares, primero son utilizadas para el consumo (50.3\%), es decir para los alimentos, el vestuario y calzado, el transporte, el equipamiento del hogar y otros.Luego, el 21.5\% es utilizado para la inversión y el ahorro, que se componen principalmente por la compra de bienes inmuebles, viviendas, maquinaria y animales. Sigue la inversión social que representa el $14.2 \%$ de las remesas, destinadas a la salud y a la educación, y finalmente, el 14.1\% de las remesas son utilizadas para el consumo intermedio, que incluye la adquisición de bienes y servicios utilizados en procesos de producción como compra de materiales y materias primas, contratación de servicios y otros. El uso que los hogares dan a las remesas muestra que los emigrantes se van para suplir las necesidades de sus familias porque no encuentran oportunidades de trabajo suficientes en Guatemala.

\section{El rol de las remesas en el desarrollo}

Numerosos académicos, políticos y miembros de organizaciones internacionales han considerado en los últimos años que los emigrantes se podrían convertir en agentes de desarrollo para su país de origen gracias al envío de remesas, ya que estas eran vistas como más efectivas que los programas de ayuda al desarrollo. Según Hein de Haas ${ }^{7}$, el debate migración-remesas/desarrollo ha polarizado los autores entre "pesimistas" y "optimistas".

Globalmente, los pesimistas argumentan que incentivar las remesas como un factor de desarrollo genera una mayor emigración de los ciudadanos y por lo tanto genera una pérdida de capital humano. También piensan que el envío regular de remesas provoca una dependencia de los hogares frente a ellas, a la vez que aumenta el consumo por bienes exportados, lo cual provoca un descenso de la

7 HAAs, Hein (2010). Migration and development, a theoretical perspective en International Migration Review, Vol 44, N²1,2010, págs.227-264. 
producción local y, por lo tanto, menos empleo y más pobreza para los que no emigran, lo cual presiona la migración. Según esta posición, la emigración y el envío masivo de remesas generan más subdesarrollo y desigualdades. Los "pesimistas" también resaltan que las remesas son en gran parte usadas para el consumo, la educación y salud, y poco invertidas o ahorradas para actividades productivas. Las comunidades receptoras de remesas, al no invertir en gastos productivos, se tornarían dependientes de las mismas. Se genera entonces según los "pesimistas" una cultura de la migración, donde dejar el país es visto como la mejor opción.

Al contrario, para los "optimistas", donde se ubican los autores neoclásicos, la migración y el envío de remesas generan desarrollo tanto para los países que expulsan como para los que reciben migrantes. Esta posición considera la migración como un mecanismo de ubicación óptima de los factores de producción, lo que aumenta el crecimiento económico. Esta perspectiva considera que al retornar a su país, los migrantes traen con ellos no sólo dinero sino nuevos conocimientos, ideas que generan una inversión productiva y que moderniza el país, disminuyendo así las desigualdades entre las naciones. Por su parte,las remesas generarían una distribución de ingresos y un mejor nivel de vida para la población y una moneda fuerte para el país. Las remesas generarían convergencia económica según los optimistas.

En el caso concreto de Guatemala, las remesas enviadas crean un vínculo entre la migración y el desarrollo del país porque representan una parte importante de la economía. Sin embargo, aunque son una fuente escencial de divisas para el país, es difícil evaluar el impacto que tienen actualmente sobre la economía y su potencial de desarrollo para las comunidades beneficiarias, porque existen pocos datos sobre los resultados concretos que generan las remesas en dicho país. No obstante, se pueden observar algunas tendencias e impactos generales en la vida del hogar, de las comunidades y del país. Si bien las remesas representan grandes volúmenes financieros que pueden tener amplios impactos económicos, el efecto de estas transferencias en la economía y para el desarrollo depende más que todo de las políticas adoptadas.

Como se mencionó anteriormente, la principal razón por la cual emigran los guatemaltecos es para suplir las necesidades financieras de sus familias. El guatemalteco que emigra tiene entonces la responsabilidad de enviar dinero a su hogar.Estas transferencias tienen capacidad de generar riqueza tanto en el hogar como en las comunidades. De hecho, el envío de remesas tiene como efecto aliviar la pobreza porque genera una mejor distribución de los recursos financieros a familias desfavorecidas en el país en la medida en que las remesas enviadas a Guatemala benefician principalmente a hogares pobres. Sin embargo, según M. Orozco ${ }^{8}$ las solas remesas no son una solución efectiva ni durable para reducir la pobreza e incrementar el desarrollo. En la mayoría de los casos, las remesas sólo representan una válvula de escape temporal a la pobreza de los hogares pero no brindan seguridad financiera

8 orozco, Manuel (2004). Remesas hacia Latinoamérica y el Caribe: cuestiones y perspectivas acerca del desarrollo en Migraciones y derechos humanos: Reunión de personas expertas, San José, 9-11 de agosto,2004,pg.92 
de manera definitiva.Las transferencias financieras alivian sobre todo la severidad de la pobreza más que el nivel de pobreza global en el país y tienen en realidad poco impacto en la reducción de la desigualdad de ingresos, desde una perspectiva estructural más que coyuntural.

Las remesas también representan un alivio al desempleo causado por la crisis económica mundial, y sobre todo por la crisis del café. Las remesas sostienen a hogares en áreas principalmente rurales pero también urbanas, permiten mejorar la vivienda y las condiciones generales de vida, y suplen la escasa cobertura social de los servicios de salud y educación. Asimismo, las remesas funcionan como una red de contención [BM,2004]. Como se ha estudiado en el texto, el crecimiento del PIB ha sido bajo en Guatemala en la última década y no ha logrado acompañar el crecimiento demográfico. Por lo tanto, las remesas son una importante fuente de ingresos y ayudan a cubrir las necesidades básicas de los hogares beneficiarios frente a la coyuntura de la crisis económica del 2008. Las remesas representan, entonces, sin duda alguna, un gran alivio para los hogares beneficiarios, pero a escala nacional, el impacto de las remesas en el desarrollo sconómico es más balanceado y difícil de comprender.

En efecto, si bien es cierto que las remesas son utilizadas en primer lugar para el consumo, parte de éstas también sirven para la construcción de nuevas viviendas, para acceder a la educación y a la salud, los cuales son factores determinantes para el desarrollo del país.Alguna proporción de las remesas también se utiliza para financiar micro empresas y empresas rurales, lo que contribuye a crear empleo.Según la encuesta de la OIM del 2005, un tercio de los hogares beneficiarios de remesas tiene su propia empresa,las remesas son la segunda fuente de financiación de éstas. El 30\% de estas microempresas tienen lugar en áreas rurales, generalmente ignoradas por las instituciones financieras guatemaltecas.

Finalmente, las remesas también permiten insertar a los países receptores en la economía mundial a través de sus migrantes. Según M. Orozco, la integración en la economía mundial, gracias a la migración activa, se hace a través de cinco áreas económicas: el turismo, las telecomunicaciones, el transporte aéreo, la transferencia de remesas y las transacciones comerciales "nostálgicas", las cuales contribuyen al crecimiento económico y al desarrollo.

Sin embargo, para que las remesas aporten de manera duradera al desarrollo del país, es necesario establecer políticas concretas, tomando en cuenta los actores involucrados, es decir los emigrantes y sus familias. Se tienen que fortalecer los vínculos entre las comunidades migrantes y sus países de origen para multiplicar el efecto de las remesas en el desarrollo. Pero si es cierto que las remesas tienen la posibilidad de fomentar el desarrollo, existen en Guatemala serios obstáculos y hasta impactos negativos producidos por el envío masivo de transferencias financieras.

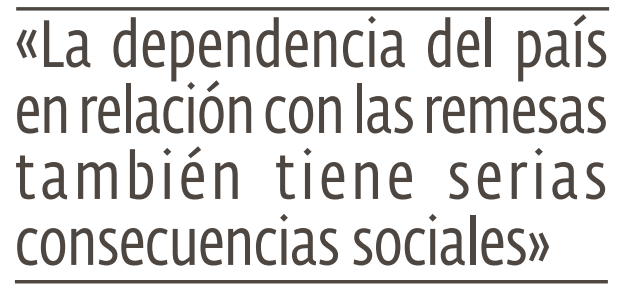


El envío de remesas a Guatemala enfrenta varios obstáculos para fomentar el desarrollo. Primero, la pobreza y sobre todo la extrema pobreza limitan el impacto de las remesas en el desarrollo. En efecto, para tener más impacto a escala nacional, las remesas tendrían que ser menos utilizadas en necesidades básicas y más en inversiones de actividades productivas. Sin embargo, si las transferencias son usadas principalmente para consumo, educación y salud, es que los hogares receptores son generalmente pobres y que antes de invertir en negocios productivos, tienen que suplir necesidades básicas. Vemos aquí que la pobreza en el país es un obstáculo para que las remesas tengan impacto en el desarrollo del país. Las remesas no han logrado romper las trampas de pobreza.

Otro problema es la incompleta cobertura de instituciones financieras en el territorio guatemalteco.Gran parte de la población, sobre todo los que poseen micro, pequeñas y medianas empresas, tienen un acceso al crédito muy limitado dado el subdesarrollo de las instituciones financieras y su escasa presencia en el campo,donde más se reciben remesas.De hecho, las instituciones financieras son actores muy relevantes para el vínculo entre remesas y desarrollo porque son ellas las que permiten que estas lleguen a los hogares,incrementando su nivel de vida y permitiendo la creación de negocios productivos.

Una tercera dificultad que se presenta en Guatemala es el alto costo que cobran los intermediarios en el envío de las transferencias y que limita el flujo de recursos que podrían ser utilizados de manera productiva. Una solución que se implementó en el país, a través de una alianza con King Express (una empresa de envío de remesas en Estados Unidos) y el banco guatemalteco Banco Industrial, consiste en que los guatemaltecos, en territorio norteamericano, puedan enviar remesas a una tasa de cambio preferencial y los hogares en Guatemala las reciban de inmediato en cualquier agencia del Banco Industrial. Además, la alianza permite que los guatemaltecos abran en este banco una cuenta de ahorro en dólares o quetzales, la moneda nacional,y tengan una tarjeta débito para tener acceso a estas cuentas en cualquier cajero. El acceso y la utilización de las remesas se vuelven por lo tanto mucho más fáciles y accesibles.

Otro riesgo resaltado por los estudios, es la dependencia que pueden generar los hogares beneficiarios de las remesas hacia estas, ya que no incentivan la búsqueda de otras fuentes de ingreso.Cuando las familias dan por sentado que recibirán este ingreso mensualmente, generalmente no crean pequeños negocios para generar nuevos ingresos. Además, existe en Guatemala el riesgo de que los jóvenes quieran emigrar para enviar dinero por lo que abandonan sus estudios. Según los estudios, los beneficios de estas prácticas parecen superar los riesgos del viaje como indocumentado a otro país. Finalmente, las escasas oportunidades laborales en el país provocan una fuga de cerebros hacia Estados Unidos y otros países “desarrollados", generando una pérdida neta de capital humano calificado para el país. Por su parte, el hecho de que la gran mayoría de las remesas provengan de Estados Unidos refuerza la dependencia de la economía guatemalteca en relación a la norteamericana. 
La dependencia del país en relación con las remesas también tiene serias consecuencias sociales. Aunque todavía no existen resultados concretos sobre las consecuencias sociales de la gran emigración guatemalteca,varios autores consideran, en teoría, que la dependencia de estos envíos genera una serie de problemas sociales. Se han realizado estudios [Hochschild, 2008] $]^{9}$, sobre el impacto de la ausencia de los padres para los niños de la familias receptoras de remesas en diferentes países que muestran que si bien éstas permiten que los hijos de los emigrantes tengan acceso a la educación, la desestructuración de las familias genera un sentimiento de abandono y soledad que perturba y puede perjudicar el desarrollo escolar y social, provocando a veces un menor rendimiento escolar y una mayor propensión a comportamientos violentos.

\section{La postura del Estadoy de otros actores frente a las remesas}

El Estado guatemalteco considera la migración como una solución a la crisis del país y como un medio potencial para fomentar el desarrollo económico a través del envío de las remesas. Por lo tanto, ha mostrado un gran interés en el asunto de la emigración de sus ciudadanos. La política migratoria estatal es vista como parte de la estrategia del desarrollo interno. El Estado guatemalteco, a través el Ministerio de relaciones Exteriores y el Ministerio de Cultura y Deporte, ha implementado varios programas para fortalecer los vínculos entre los migran-

9 ISAKSEN,Lise; UMA DEVI,Sambasivan; HOCHSCHILD, Arlie Russell. Global care crises: a problem of capital, care chain or commons. En: American Behavioral Scientist. Volumen 52, No.3. Noviembre 2008. Pp.405 - 425 tes y sus comunidades de origen como ferias comerciales y eventos culturales. Se ha creado por ejemplo el Programa Trabajadores(as) Agrícolas Temporales a Canadá, a través del cual, guatemaltecos son enviados a trabajar a dicho país por un tiempo predeterminado. La vicepresidencia también trabaja en cooperación con la OIM para vincular las remesas a proyectos comunitarios, esto daría por resultado que el gobierno duplicaría el monto de remesas enviado por los emigrantes. Igualmente, el país modernizó las regulaciones en cuanto a las actividades en moneda extranjera y existen dos instituciones que recopilan los datos sobre las remesas: el Banco Central y la Unidad de Inteligencia Financiera de la Superintendencia de Bancos. Sin embargo, todavía no existen datos sobre los resultados de estos programas.

Como parte de la política migratoria, se diseñó el Plan de Gobernabilidad de las Migraciones que consiste en proveer atención a los migrantes en el exterior a través de acuerdos con el gobierno de Estados Unidos para facilitar los servicios de salud y educación,y el fortalecimiento de los consulados para proveer asistencia legal y humanitaria a los migrantes. También se establecieron programas para facilitar el acceso a servicios de salud y educación para las familias de los migrantes en Guatemala, financiados por las remesas.

Para aliviar el obstáculo del costo de las transferencias, se implementaron políticas para reducir los costos de envío de las remesas y bancarizar a los migrantes en el exterior y a las familiares beneficiarias. Además, se intenta potenciar el impacto de las remesas sobre el ahorro a través el fortalecimiento de infraes- 
tructura financiera en el país para captar las transferencias financieras que en la práctica de realizan muchas veces por canales informales.

Otro programa novedoso para potenciar el desarrollo a través de las remesas llevado a cabo por la Oficina de la OIM de Guatemala es la creación de un Sistema Nacional de Fondos Comunitarios. Este fondo tiene como misión proporcionar nuevas oportunidades de desarrollo a través del envío de dinero para las comunidades de proveniencia de los migrantes en Estados Unidos. La idea es que los vecinos y los migrantes de una misma comunidad compartan un mismo mecanismo financiero de cuentas bancarias personales que permita acumular los ahorros de la comunidad en una sola cuenta comunitaria. Los vecinos y migrantes de una comunidad se pueden convertir en socios de un Fondo Comunitario y cada usuario de la cuenta puede poner sus recursos financieros en una cartera de créditos para invertir en pequeñas empresas o en obras de infraestructura o servicios sociales para la comunidad.

\section{Conclusiones}

Las remesas representan una parte importante en la economía guatemalteca, sobre todo en periodos de crisis. Sin embargo, no son suficientes en sí para generar desarrollo. En efecto, sí alivian la situación económica de millones de hogares y los ayudan a subsistir, permitiéndoles tener acceso a servicios básicos de salud y educación; sin embargo no inciden en una baja global de los niveles de pobreza o de desigualdad. Para potencializar el efecto de las remesas sobre el desarrollo,son necesarias políticas públi- cas y una mayor coordinación entre las instituciones que recopilan datos sobre estas operaciones para que se haga un análisis más profundo y puedan diseñarse políticas adecuadas para transformar las remesas en desarrollo. También es muy útil promover eventos y cooperación entre instituciones financieras de Guatemala y de los países donde se encuentran los migrantes. Otra medida que tendría efecto sería facilitar el proceso burocrático para montar microempresas y que haya más presencia de instituciones financieras en el campo.

Finalmente, si las remesas pueden fomentar el desarrollo cuando se establecen políticas para multiplicar sus efectos, se plantea la pregunta de la legitimidad de utilizar estas transferencias, producto del trabajo de ciudadanos que tuvieron que dejar su país y sus hogares porque no encontraban en el territorio nacional las condiciones de seguridad, económicas y humanitarias para vivir en buenas condiciones. La migración y el envío de dinero muestran que el gobierno guatemalteco ha fallado en el desarrollo interno del país, obligando, en cierta medida, la emigración de sus ciudadanos que buscan mejores oportunidades. Por lo tanto, la responsabilidad de generar desarrollo no debe apoyarse completamente en los ciudadanos en el exterior.Los migrantes se vuelven un instrumento económico y una válvula de escape y suplen las deficiencias del Estado.El hecho de que el 10\% de una población tenga que emigrar para que sus familias tengan mejores condiciones de vida oculta efectos sociales nefastos como la desintegración familiar y la ausencia de los padres, que a su vez causan problemas en la escolaridad de 
los jóvenes, socialización en pandillas y delincuencia general.

\section{Bibliografía}

\section{Artículos académicos}

ISAKSEN, Lise; UMA DEVI, Sambasivan; HOCHSCHILD, Arlie Russell (2008). Global care crises: a problem of capital, care chain or commons. En:American Behavioral Scientist. Volumen 52, No.3.

HAAS, Hein (2010). Migration and development, a theoretical perspective, en International Migration Review, Vol 44, $\mathrm{N}^{\circ} 1$, págs.227-264.

LOZANO, Victor (2005). Encuesta Nacional sobre emigración internacional de guatemaltecos 2002-2003" en Población y Salud en Mesoamérica, Vol.2, $\mathrm{N}^{\circ} 2$, art.4, ene-jun 2005, 36 págs. Disponible en: redalyc.uaemex.mex

MONTOYA ZAVALA, Erika (2006). Experiencias internacionales en el uso productivo de las remesas, en Migración y Desarrollo, 2006 Primer Semestre, págs.131-150

TEDESCO, Laura (2008). Remesas, Estado y desarrollo, en FRIDE, Documento de trabajo $\mathrm{n}^{\circ} 72$, Noviembre 2008, 24 págs. Disponible en: www.fride.org

\section{Informes oficiales}

BANCO MUNDIAL. Análisis del corredor de remesas Estados Unidos-Guatemala, 24 de abril de 2006, 87 págs. Disponible en: siteresources.worldbank.org/GUATEMALAINSPANISHEXT/.../corredorremesaSEEUUGuatemala.pdf

MARTINEZ PIZARRO, Jorge (2005). La eXperiencia de la CEPAL en los estudios sobre remesas: lecciones y evidencias" (Semi- nario internacional: Problemas y desafíos de la migración y el desarrollo en América), abril 2005, Cuernavaca, Morelos, 28 págs. Disponible en: www.remesasydesarrollo. org/uploads/media/la_experiencia_de_la_ cepal.pdf

OIM, (2002). Programa Nacional de Fondos Comunitarios para Guatemala: Inversiones Conjuntas entre Vecinos y Migrante, en Cuadernos de Trabajo Sobre Migración, $\mathrm{n}^{\circ} 11$, Guatemala, 2002, 36 págs. Disponible en: www.oim.org.gt/.../Cuaderno\%20de $\% 20$ Trabajo\%20No.\%2011.pdf

OIM (2003). Encuesta Nacional sobre Emigración Internacional de Guatemaltecos. Resultados Definitivos, en Cuadernos de Trabajo Sobre Migración, ${ }^{\circ}{ }^{15}$, Guatemala, 2003, 138 págs. Disponible en: www.oim. org.gt/.../Cuaderno\%20de\%20Trabajo\%20 No.\%2015.pdf

OIM (2003). Encuesta Nacional sobre Remesas Familiares, en Cuadernos de Trabajo Sobre Migración, $\mathrm{n}^{\circ} 17$, Guatemala, Agosto de 2003, 104 págs. Disponible en: http://www.oim. org.gt/documentos/Cuaderno\%20de $\% 20$ Trabajo\%20No.\%2017.pdf

OIM (2006). Encuesta sobre Remesas 2006. Inversión en Salud y Educación" en Cuadernos de Trabajo Sobre Migración, $\mathrm{n}^{\circ} 23,145$ págs. Disponible en: www.oim.org.gt/.../Cuaderno\%20de\%20Trabajo\%20No.\%2023.pdf

OROzCo, Manuel (2004). Remesas hacia Latinoamérica y el Caribe: cuestiones y perspectivas acerca del desarrollo, en Migraciones y derechos humanos: Reunión de personas expertas, San José, 9-11 de agosto, 2004, pgs.61-133. Disponible en: https:// www.bmi.gob.sv/pls/.../63AD08F19B1EF9 DBE040558CE3C9744B

RATHA, Dilip, MOHAPATRA, et al.(2007). Tendencias de las remesas en 2007 en Migración 
y Desarrollo - Nota informativa N. 03, 29 de noviembre de 2007, 8 págs. Disponible en:

siteresources.worldbank.org

\section{Sitios web}

Sitio de la Oficina de la oim en Guatemala:

http://www.oim.org.gt/

Sitio oficial de la migración en Guatemala:

http://www.migracion.gob.gt 